\title{
ACCIÓN Y REACCIÓN EN ÉPOCA DE PANDEMIA: LA UNIVERSIDAD ARGENTINA ANTE LA COVID-19
}

\author{
Ana Fanelli \\ Doctora en Economía. Investigadora principal del Consejo Nacional \\ de Investigaciones Científicas y Técnicas (Conicet) de Argentina, \\ en el Centro de Estudios de Estado y Sociedad (CEDES). \\ anafan@cedes.org \\ Mónica Marquina \\ Doctora en Educación Superior. Profesora e investigadora \\ en la Universidad Nacional de Tres de Febrero (Nifede), \\ Conicet, Universidad de Buenos Aires (FFyL). \\ momarquina@gmail.com \\ Marcelo Rabossi \\ Doctor en Educación por la University at Albany (SUNY). Profesor \\ e investigador en la Universidad Torcuato Di Tella, Argentina. \\ mrabossi@utdt.edu
}

\section{Introducción}

La llegada de la COVID-19 pareciera cambiar algunos paradigmas. Uno de ellos, en el sector educativo, el cual, sin respiro, se vistió de modalidad a distancia. Aun cuando las lecciones que estamos aprendiendo al andar resultarán positivas, difícilmente provoquen un cambio de 180 grados. En las universidades, es probable que la virtualidad complemente a la presencialidad, pero sin reemplazarla.

\section{G6 En las universidades, es probable que la virtualidad complemente a la presencialidad, pero sin reemplazarla

Las más de 25.000 instituciones, que conforman el sistema global de educación superior, reaccionaron rápidamente con el fin de encontrar una solución para los cerca de 200 millones de estudiantes que poblaban sus aulas antes de la pandemia. Este rá- 
pido giro, desde lo presencial hacia lo virtual, no encontró a todas las instituciones de la misma forma. En América Latina, en promedio, algo menos del 50\% de los hogares cuenta con acceso a la red, aunque podría suponerse que en aquellos con estudiantes universitarios sería un poco mayor. La tasa de conectividad muestra una asimetría de amplio espectro entre países. Mientras que en Bolivia y El Salvador apenas el 20\% de los hogares está conectado, en Argentina y Chile, alrededor de ocho de cada diez hogares accede a internet.

Argentina, como otros países de América, tuvo la posibilidad de advertir cómo el virus se propagaba sin tregua por Asia y Europa, a partir de febrero. El primer caso confirmado de coronavirus sucedió el 3 de marzo, y la primera persona fallecida, diez días después. El 20 de marzo el gobierno dispuso el "aislamiento social, preventivo y obligatorio" para toda la población residente en el país, suspendiendo toda actividad no esencial, el transporte interno y externo; así como el cierre de fronteras. Esta situación permanece hasta la actualidad, con una lenta apertura a algunas actividades, que no incluyen a la educación.

Desde lo económico, la actual situación tendrá un mayor impacto en aquellos países con menores recursos y, a su interior, entre la población más vulnerable. Organismos internacionales anticipan una retracción del 3\% del PBI mundial durante 2020, la mayor desde la depresión de la década del treinta. Para la Argentina, acostumbrada a los vaivenes económicos, significará acumular un tercer año consecutivo de caída económica. Producto de una cuarentena impuesta desde fines de marzo a casi la totalidad del aparato productivo del país, la recaudación tributaria exhibe un fuerte retroceso. Como consecuencia, las universidades deberán enfrentar un golpe de importancia. Recordemos que el sec- tor público ofrece educación universitaria absolutamente gratuita a casi el $80 \%$ de los estudiantes de pregrado. Las 57 universidades estatales dependen casi exclusivamente del erario público para seguir funcionando. El sector privado universitario, en donde el cobro de aranceles representa alrededor del 90\% de los ingresos totales, enfrentará un estrés financiero cuyas consecuencias no son posibles de prever.

En este contexto, analizamos los principales efectos que, hasta mayo de 2020, se aprecian en las funciones esenciales de la actividad universitaria argentina: la enseñanza, la investigación y la colaboración de la universidad a la sociedad en la solución de los problemas planteados por la emergencia sanitaria.

\section{Efectos sobre la enseñanza}

El cambio imprevisto a la enseñanza online, en la vasta mayoría de las universidades argentinas, cuenta con un antecedente que la diferencia de otros países de la región. Si bien desde el inicio del siglo XXI, la educación virtual universitaria comenzó a expandirse de manera notable, con algunas universidades muy destacadas en estos desarrollos, en el año 2017 el sistema universitario en su conjunto acordó un marco legal para asegurar condiciones institucionales de calidad para el desarrollo de esta modalidad. Desde entonces, además de que cada programa a distancia — sea de pregrado o posgrado - tenga que atravesar un proceso de acreditación específico, las instituciones que deciden contar con ofertas educativas a distancia, total o parcial, deben crear su Sistema Institucional de Educación a Distancia (SIED), el cual debe ser validado por la Secretaría de Políticas Universitarias, previa evaluación favorable de la Comisión Nacional de Evaluación y Acreditación Universitaria. 
A la fecha, casi 100 de las 135 universidades han presentado su SIED. Si bien esta regulación fue pensada para dar marco a los programas que fueron diseñados a distancia —en la mayoría de las instituciones una porción menor de la oferta total de formación-, hoy constituye una base estructural clave para afrontar la emergencia, que diferencia al nivel universitario de otros niveles educativos. La mayoría de las universidades estatales, a partir de la instauración de la cuarentena y asumiendo que se trata de una situación transitoria y de emergencia, comenzaron a trabajar de manera veloz para disponer sus entornos virtuales en una gran parte de las materias programadas, al menos en las que no está implicada la formación práctica, más compleja de diseñar en formato virtual. Además, asumiendo que este cambio podría profundizar las ya existentes inequidades, el gobierno firmó un compromiso con las empresas prestadoras de servicios de telefonía e internet para liberar el uso de datos móviles en el acceso de los estudiantes a las plataformas educativas de las 57 universidades públicas. A la fecha, la mayoría dicta clases virtuales, incluyendo defensas de tesis.

\section{C6 Asumiendo que este cambio podría profundizar las ya existentes inequidades, el Gobierno firmó un compromiso con las empresas prestadoras de servicios de telefonía e internet para liberar el uso de datos móviles en el acceso de los estudiantes a las plataformas educativas}

No obstante, la situación varía en función de las características y posibilidades de cada institución. La Universidad de Buenos Aires (UBA), la más grande del país en cantidad de alumnos, estableció un nuevo calendario académico que pospuso su inicio

de abril a junio. Aun reconociendo, en función de la gran diversidad de unidades académicas y programas, que cada facultad puede decidir establecer actividad virtual, la UBA aprobó una normativa que sostiene que la presencialidad de las clases y su correspondiente evaluación es la opción formal autorizada, bajo argumentos de responsabilidad y calidad. Esta decisión generó algunas tensiones en el sistema universitario. Otras universidades públicas, con una larga historia y reconocimiento, como las Universidades Nacionales de Córdoba, de la Plata, de Rosario, de Cuyo, Litoral, y posteriormente la gran mayoría de las instituciones de más reciente creación, ratificaron las clases virtuales como vías para sostener la actividad académica durante la cuarentena, plasmando la decisión, además, en una declaración conjunta del Consejo Interuniversitario Nacional.

Por su parte, el sector privado siguió una decisión similar. Las universidades privadas comenzaron a dictar sus cursos online, especialmente en aquellas materias con contenido teórico, esperando retomar la parte práctica cuando se regrese a la presencialidad. Si bien todas las universidades adoptaron la misma estrategia, algunas ya contaban con experiencia previa en cursos a distancia y en el uso de campus virtuales para la actividad de enseñanza. En estos casos, el paso a la enseñanza remota tuvo lugar tras una rápida capacitación a los docentes y la incorporación y difusión del uso de programas para el dictado de los cursos. Aquellas con menor experiencia en la enseñanza online, enfrentan actualmente un desafío más acuciante, pues ofertaron cursos remotos sin contar con la infraestructura y con el personal capacitado para su uso. En general, dichas políticas se han enmarcado en los denominados "comités de crisis" de cada institución, con el objetivo de dar una rápida respuesta a la contin- 
gencia. En estos ámbitos, además de docentes y autoridades, se ha incluido al personal administrativo para continuar con sus labores de manera remota.

La mayoría de las universidades privadas vieron disminuidos sus ingresos, sobre todo, las que por su perfil cuentan con un alumnado perteneciente a estratos socioeconómicos menos acomodados. Ante este hecho, se ha buscado priorizar el pago de salarios al personal y todo lo referente a la mejora de los procesos de conectividad. Como consecuencia, las nuevas inversiones en infraestructura se encuentran por ahora postergadas. Pero, la situación podría llegar incluso a afectar la sostenibilidad financiera de estas instituciones, ya que en algunos casos los alumnos han manifestado no estar dispuestos a pagar los aranceles durante la etapa virtual.

La disminución en la cantidad de estudiantes internacionales es otro dato a tener en cuenta, ya que afecta al incipiente proceso de internacionalización de la educación superior en la Argentina. Tanto en las universidades públicas como en las privadas, se ha cancelado la movilidad de estudiantes internacionales para lo que resta del año. Más de la mitad de los estudiantes internacionales regresó a sus países o nunca arribó a la Argentina. En promedio, se estima que solo uno de cada tres permanece en el país de un total de más de 90.000 alumnos extranjeros. En algunos casos, las instituciones privadas les han ofrecido el cursado bajo modalidad a distancia, lo cual es aceptado solo parcialmente por los estudiantes internacionales.

La extensión de la situación de emergencia llevó a que los consejos de rectores, de las universidades nacionales y de las privadas, comenzaran a discutir sobre los pasos para asegurar la validez de la actividad académica virtual llevada adelante, sobre todo, en cuanto a las evaluaciones, en virtud de que se trata de carreras cuyo reconocimiento y validez oficial se ha obtenido bajo la modalidad presencial. Sin embargo, la propia normativa ya mencionada, que regula hoy la educación universitaria a distancia a nivel institucional y de programas, posibilita que toda carrera bajo la modalidad presencial pueda ofrecer hasta un $50 \%$ de sus actividades curriculares bajo la modalidad a distancia, quedando de esta manera cubierta la actual situación excepcional y de emergencia por un año e incluso más.

Por otra parte, comienzan a escucharse quejas, reclamos y hasta posicionamientos gremiales sobre la situación del docente universitario ante esta situación excepcional. No obstante, se observa que se está aprendiendo sobre la marcha, y que en muchos casos el formato de equipo de cátedra, en el que trabajan profesores consolidados con jóvenes docentes, está ayudando a un intercambio inusual en el que se conjugan diferentes tipos de saberes. Pareciera que la comunicación, el intercambio entre docentes y entre instituciones son una buena salida frente a esta circunstancia. $Y$ es bueno que así sea, porque es probable que al iniciarse las clases presenciales sean los más jóvenes los que tengan que iniciar el dictado presencial.

\section{G6 El formato de equipo de cátedra, en el que trabajan profesores consolidados con jóvenes docentes, está ayudando $a$ un intercambio inusual en el que se conjugan diferentes tipos de saberes}

\section{פ}

Finalmente están las y los estudiantes. Mucho se lee por estos días sobre la base desigual de la virtualidad en términos sociales, bajo el argumento de 
que la opción a distancia debería cumplir con una condición de equidad o inclusión que no tiene necesariamente una idealizada formación presencial. Sin duda, en la actual emergencia las inequidades se exacerban, por lo que muchas instituciones han tomado esta situación como prioritaria, en particular, cuando sus estudiantes no tienen acceso a conectividad. Pero, hay una realidad cada vez más generalizada: los y las estudiantes piden estar en contacto y están, en general, dispuestos a trabajar codo a codo con sus docentes para seguir aprendiendo en esta difícil situación.

\section{Investigación, transferencia de conocimientos y capacidades a la sociedad}

Un rasgo peculiar de la pandemia, al impactar como factor exógeno sobre el sector universitario, es que por su misma naturaleza reforzó la percepción social sobre la relevancia del quehacer científico, y el papel de las universidades como productoras de conocimiento y generadoras de soluciones frente a este drama sanitario.

En cuanto a la actividad de investigación, las universidades mostraron una rápida capacidad de respuesta frente a las acciones impulsadas por el gobierno, a través de la denominada "Unidad Coronavirus" dependiente del Ministerio de Ciencia, Tecnología e Innovación. La creación de esta unidad buscó promover el uso de las capacidades de desarrollo de proyectos tecnológicos, recursos humanos, infraestructura y equipamiento para realizar tareas de diagnóstico e investigación sobre la COVID-19. Dentro de sus acciones se encuentra la actividad de $\mathrm{I}+\mathrm{D}$, a través del concurso de fondos competitivos para la investigación. En particular, la Agencia Nacional de Promoción de la Investigación, el Desarrollo Tecnológico y la Innovación realizó una "Convocatoria
Extraordinaria Ideas-Proyecto COVID 19", para la asignación de fondos a proyectos por un valor total de 5 millones de dólares con financiamiento del BID. Dado el bajo nivel de inversión destinado a la actividad de I+D que acompañó la desaceleración de la economía desde 2018, esta convocatoria implicó un estímulo muy importante para los grupos de investigación. Cabe al respecto tener en cuenta que los montos a asignar por proyecto ascendieron a $100 \mathrm{mil}$ dólares. La respuesta del sector universitario a este concurso de fondos fue notable. En el corto lapso entre el 27 de marzo y el 10 de abril de 2020, se presentaron 900 ideas-proyecto (IP). Dentro de estas IP estuvieron presentes, con uno o más proyectos por universidad, 46 universidades nacionales, 2 provinciales y 13 privadas. De estas 900 IP, fueron seleccionadas 64 iniciativas, entre las que se encuentran aquellas presentadas por 17 universidades nacionales y una privada.

Además de esta respuesta del mundo universitario para producir nuevo conocimiento vinculado con la pandemia, un grupo de universidades puso sus recursos humanos, conocimientos científicos y capacidad instalada para ofrecer distintos tipos de soluciones frente a la emergencia sanitaria. Se destacan, en particular, las acciones desarrolladas por las universidades con carreras de salud y hospitales. A modo de ejemplo, los estudiantes de medicina de la Universidad de Buenos Aires se ofrecieron como voluntarios para colaborar con distintas acciones emprendidas por el gobierno, tales como participar en campañas de vacunación contra la gripe, realizar el primer control de los pacientes o el seguimiento de estos en sus casas. La Universidad Nacional de Quilmes y la Universidad Nacional de La Plata se incorporaron a los centros encargados de realizar pruebas para el diagnóstico de coronavirus. En la Universidad Nacional del Litoral, un grupo 
de investigadores trabajará conjuntamente con las empresas Cellargen Biotech SRL y Biotecnofe SA, para desarrollar proteínas que puedan ser utilizadas en vacunas y kits de diagnóstico para prevención y monitoreo del coronavirus. La Universidad Nacional de Rosario, en alianza con una empresa privada, financió proyectos para desarrollar respiradores artificiales a bajo costo. La Universidad Austral está construyendo el Hospital Solidario COVID Austral, de alta complejidad y atención gratuita para personas sin acceso a la salud.

Las universidades también colaboraron en el reclutamiento de voluntarios para la atención de pasajeros recién llegados en hoteles o en barrios de emergencia, así como el acompañamiento a adultos mayores. Asimismo, prestaron servicios en la producción de insumos en escasez, elementos de protección y equipamiento hospitalario; y en la disposición y acondicionamiento de sus hospitales universitarios.

En suma, la comunidad universitaria y, en particular, sus investigadoras e investigadores se alinearon con los requerimientos del sistema de salud para hacer frente a una situación inédita en el país y en el mundo.

\section{Conclusiones}

La pandemia fue un factor exógeno a las organizaciones universitarias argentinas que desencadenó cambios en sus principales funciones con una velocidad no experimentada antes. Estas primeras acciones de la comunidad universitaria revelan su capacidad de adaptación frente a las amenazas y desafíos que planteó la emergencia sanitaria. Las respuestas revelaron también la heterogeneidad de las instituciones universitarias en términos de sus recursos y experiencias previas con la educación remota. Además de una probable brecha digital entre los estudiantes, enfrentamos también una brecha digital entre las instituciones universitarias.

\section{C6 La pandemia fue un factor exógeno a las organizaciones universitarias argentinas que desencadenó cambios en sus principales funciones con una velocidad no experimentada antes}

\section{g}

Todavía resulta difícil identificar qué transformaciones permanecerán una vez superada la pandemia. Los cambios en la función de enseñanza han sido promovidos desde las cúspides de decisión universitarias. Desconocemos en qué medida las y los docentes han logrado adaptarse exitosamente a estas transformaciones. Tampoco existe información sobre resultados de aprendizaje en esta nueva modalidad de enseñanza. Queda aún por resolver qué pasará con las evaluaciones si la cuarentena se prolonga, tal como se prevé. En la planificación del "día después" las universidades deberán enfrentar otro reto: la probable reducción de sus presupuestos en términos reales, en el marco de la crisis económica que ya atraviesa la Argentina y que se profundizará a futuro.

Sin embargo, en términos de externalidades positivas, esta pandemia ha permitido consolidar la legitimidad de la función investigación y social de la universidad de cara a la comunidad. En cuanto a la función de enseñanza, puede dejar una experiencia que será fundamental analizar. La virtualidad como complemento de la educación presencial podría ayudar a repensar nuevos modelos pedagógicos, rediseñar el currículum y otros asuntos que siempre han estado pendientes en la educación universitaria argentina. 